\title{
BMJ Open Neuroimaging in the effect of transcranial magnetic stimulation therapy for patient with depression: a protocol for a coordinate-based meta- analysis
}

Yongyan Deng, Wenyue Li, Bin Zhang

To cite: Deng Y, Li W, Zhang B. Neuroimaging in the effect of transcranial magnetic stimulation therapy for patient with depression: a protocol for a coordinate-based meta-analysis. BMJ Open 2020;10:e038099. doi:10.1136/ bmjopen-2020-038099

- Prepublication history and additional material for this paper is available online. To view these files, please visit the journal online (http://dx.doi.org/10.

Received 28 February 2020 Revised 26 August 2020 Accepted 02 September 2020 1136/bmjopen-2020-038099).

\section{ABSTRACT}

Introduction As a prevalent psychiatric disease, depression is a life-threatening mental disorder that may cause work disability and premature death. Transcranial magnetic stimulation (TMS) is a non-invasive neuromodulation procedure, which has been reported to have a significant effect on antidepressant treatment in recent years. However, the parameters of TMS for depression that can produce the best clinical benefits remain unknown. In the present study, we will evaluate the effect of TMS treatment for depression from the perspective of functional neuroimaging by performing a meta-analysis based on included studies.

Methods and analysis Two independent reviewers will search published studies in the following five databases: PubMed, Web of Science, Embase, China National Knowledge Infrastructure and WANGFANG DATA from inception to 1 June 2020. Then we will select studies according to predesigned inclusion and exclusion criteria. After extracting data from included studies, activation likelihood estimation will be applied to data synthesis. Any disagreement will be checked by the third reviewer who will also make the final decision.

Ethics and dissemination This work does not require ethics approval as it will be based on published studies. This review will be published in peer-reviewed journals. PROSPERO registration number CRD42020165436.

\section{Check for updates}

\section{(c) Author(s) (or their} employer(s)) 2020. Re-use permitted under CC BY-NC. No commercial re-use. See rights and permissions. Published by BMJ.

Psychiatric and psychological Neuroimage Lab (PsyNI Lab), Affiliated Brain Hospital of Guangzhou Medical University, Guangzhou, Guangdong, China

Correspondence to

Dr Bin Zhang;

zhang.bin845@foxmail.com

\section{INTRODUCTION}

Depression is a highly prevalent, long-lasting and recurrent mental disorder, from which over 300 million people are suffering at the global level. ${ }^{1}$ As one of the most debilitating mental illnesses, depression is a strong predictor of work disability and premature death, related to cognitive impairment and long-term memory dysfunction. ${ }^{23}$

Although there are a range of antidepressant therapies, about a third of patients do not respond to any pharmacotherapy or psychotherapy treatment and instead remain symptomatic and functionally
Strengths and limitations of this study

- Equipped with advanced data processing techniques, this could be the first systematic review and meta-analysis that uniquely explore brain functional changes after transcranial magnetic stimulation for depression in neuroimaging.

- Quantitative evaluation will be applied to data processing in this meta-analysis.

- Only studies in English and Chinese will be included, which may cause language bias.

- A customised checklist will be used to evaluate the quality of the included studies.

- There may be heterogeneity among the included studies due to the variety of participants' characteristics and study design.

impaired. ${ }^{45}$ Moreover, cognitive impairment is also frequently observed in patients with depression, and the relationship between depression and cognition is bidirectional. Both depressive mood and cognition may be associated with impaired brain plasticity, presenting with impaired cortical activity and the dysregulation of the connectivity. ${ }^{6}$ On the other hand, some studies performed singlepulse and paired-pulse tanscranial magnetic stimulation (TMS) both before and after the repetitive TMS (rTMS), in order to objectively assess any change of cortical excitability and found significant hemispheric asymmetry in MDD (Major Depressive Disorder); that is, compared with the right hemisphere, the excitability of both inhibitory and facilitatory inputs of the left hemisphere was found to be reduced. ${ }^{7}$

Current clinical treatments for resistant depression usually involve progressively empirical attempts for more complex forms of medication therapy, which may diminish the likelihood of achieving remission as the 
number of failed attempts increase. ${ }^{4}$ TMS, a method of stimulating the brain through the scalp and skull without causing any pain at the surface, can be a safe and effective option for depression treatment. ${ }^{48}$ In particular, rTMS, a TMS technique commonly used in clinical treatment, provides a long-lasting therapeutic benefit on mood and cognitive performance of depression related to its intrinsic ability to modulate cortical excitability and connectivity of the areas underlying mood and affect regulation by causing plastic remodelling of the synaptic connections. ${ }^{69}$ Limited to the attenuation with distance from the coil surface and the site of stimulation without focal point, technical parameters of TMS produce the best clinical benefit remain unknown. ${ }^{810}$ Therefore, it is essential to evaluate neuropsychiatric effects when applying several sets of frequently used coil placements, coil distances and other parameters (eg, frequency, intensity, duration, rate, time per session, pulses per condition and total stimulation sessions) of TMS. Functional neuroimaging provides a distinctive perspective to explore neuropsychiatric change after TMS treatment.

In recent years, researchers have begun to focus on the effect of TMS therapy on functional neuroimaging changes for patients with depression. A recent study has demonstrated that patients' blood-oxygen leveldependent signal power was significantly reduced in clusters localised in the medial prefrontal cortex, caudate, putamen, thalamus and anterior cingulate after receiving TMS antidepression treatment, targeted at the dorsolateral prefrontal cortex (DLPFC). ${ }^{11}$ Another trial has demonstrated higher significant functional connectivity to the left ventromedial prefrontal cortex in a left-lateralised set when stimulating the dorsomedial prefrontal cortex (DMPFC) for those patients with depression responding to TMS treatment, proving that DMPFC may be a promising and complementary target different from DLPFC, which has been recognised as a typical region of TMS therapy for depression. ${ }^{12-14}$

However, the results of these studies are inconsistent. There is no systematic review or meta-analysis that summarises the effect of antidepression TMS treatment on brain functional changes. Therefore, synthesising previous studies may offer a better understanding of brain functional mechanisms of TMS for depression.

\section{OBJECTIVE}

In the present meta-analysis, we will comprehensively assess previous studies to evaluate the effect of TMS treatment on brain functional changes for patients with major depression.

\section{METHODS AND ANALYSIS}

Preferred Reporting Items for Systematic Reviews and Meta-Analyses Protocols will be set as a guide book for the protocol (see online supplemental material). ${ }^{15}$

\section{ELIGIBILITY CRITERIA}

The inclusion and exclusion criteria will be revealed as follows.

\section{Study types}

Case-control studies, cohort studies and randomised controlled trials will be included, whose original functional neuroimaging data are available to extract. While case reports, systematic reviews, meta-analyses, letters and other second-hand studies will be excluded.

\section{Study characteristics}

We will include peer-reviewed original studies. Reviews, meta-analyses, anecdotal case reports, conference proceedings and unpublished theses will be excluded. We will also exclude studies with subjects that overlap and those that employed the same task. All appropriate studies published before 1 June 2020 will be included. Language will be restricted to English and Chinese.

\section{Study design}

We aim to investigate patients' brain functional activity and functional connectivity changes after receiving antidepressant TMS treatment. Functional neuroimaging data before and after treatment are necessary. Both resting-state and task neuroimaging studies will be included. Only studies that reported coordinates in either the Talairach or Montreal Neurological Institute (MNI) space will be included. Studies that investigate structural imaging changes will be excluded. Also, we will include peer-reviewed original articles of clinical trials investigating the effects of TMS as monotherapy or in combination with other treatments in patients with depression. Both controlled studies (compared with sham treatments) and uncontrolled studies (without sham treatments) will be included.

\section{Participants}

Patients with depressive disorder diagnosed by ICD-10 (International Classification of Diseases) or DSM-5 (The Diagnostic and Statistical Manual of Mental Disorders) will be included. Both unipolar depression and bipolar depression will be included. Mixed studies that had participants coinitiate another form of treatment, such as different pharmacotherapy or psychotherapy treatment will be included. Patients limited to a specific subtype of depression (eg, postpartum depression, vascular depression or poststroke depression) or in which a major depressive episode was a secondary diagnosis (eg, fibromyalgia and major depression) will be excluded. ${ }^{16}$ The race and age of participants will not be restricted.

\section{Exposure}

The studies that only applied TMS treatment for patients with depression disorder rather than other antidepressant treatment will be included.

\section{Outcome measures}

The primary outcomes of the included studies should be depression patients' functional brain's alterations after TMS treatment. Brain functional outcomes are related to the 
Table 1 Searching strategy

\begin{tabular}{ll}
\hline 1.1 PubMed searching strategy & $\mathbf{1 . 2}$ CNKI searching strategy \\
\#1 depression (MeSH Terms) & \#1抑郁症 (主题词) \\
\#2 deprression (All Fields) & \#2磁共振成像 (主题词) \\
\#3 deprress (All Fields) & \#3 MRI (主题词 ) \\
\#4 \#1OR \#2 OR \#3 & \#4 \#2 OR \#3 \\
\#5 Transcranial magnetic stimulation (MeSH Terms) & \#5经乑磁刺激 (主题词) \\
\#6 Transcranial magnetic stimulation (All Fields) & \#6 TMS (主题词) \\
\#7 \#5 OR \#6 & \#7 \#5 OR \#6 \\
\#8 magnetic resonance imaging (MeSH Terms) & \#8 Final search terms: \#1 AND \#4 AND \#7 \\
\#9 magnetic resonance imaging (All Fields) & \\
\#10 MRI (All Fields) & \\
\#11 \#8 OR \#9 OR \#10 & \\
\#12 Final search terms: \#4 AND \#7 AND \#11 & \\
\hline
\end{tabular}

CNKI, China National Knowledge Infrastructure .

whole brain functional activity and functional connectivity, whose coordinates in either the MNI or Talairach space are reported in included studies. Functional MRI data (eg, software, statistical threshold, correction step, task and source of coordinate) and the characters of the region of interest (eg, coordinates in MNI or Talairach space, cluster size and t-statistic) will be extracted from the included studies.

The secondary outcome pertaining to these studies may be extracted as mean change in scores for depression and TMS treatment parameters. We extracted the data on the levels of depression according to the most commonly used scales (eg, The Hamilton Depression Rating Scale and the Beck Depression Inventory) in the included studies. As for TMS parameters, related frequency, intensity, duration, rate, time per session, pulses per condition and total stimulation sessions of treatment will be extracted.

\section{Searching strategy}

The relevant articles will be identified by searching the electronic bibliographic databases: PubMed, Web of Science, EMbase, Wanfang Data, China National Knowledge Infrastructure (CNKI). Medical subject heading terms will be used. The searching strategies of PubMed (English) and CNKI (Chinese) are shown in table 1 as examples to replicate for the other databases.

\section{Selection process}

We will use EndNote V.X7 software (Thomson Reuters, New York, USA) to manage the search results and perform screening. After removing duplicates, two independent reviewers will examine the titles and abstracts of the identified studies and will exclude those which are irrelevant. Then full articles will be obtained and checked again in more detail for the final inclusion. Any conflict will be solved by discussion, in which the third reviewer will participate. Final selection will be checked and determined by the third reviewer. This process will be completed on 10 July 2020 .

\section{Data collection}

Data will be extracted independently by two reviewers from the selected studies based on standardised pilot-tested data forms. The third reviewer will check and decide the final extraction in case of any disagreement. Extracted data are as follows:

Study characteristics: study name, author(s), year of publication, study location and study setting.

Study design: treatment parameters, mean treatment duration, imaging modalities, software.

Study population: basic demographics of participants, methods of depression diagnosis and other relevant information.

Details of the interventions and comparators: relevant outcome data for the primary outcome (sample size, numbers of coordinates reported, threshold, peak).

All measures will be collected before and after antidepressant TMS treatment. If there is any missing data, we will try to contact the author. We will not record data missing until we wait for a week. Completion time for this process will be tentatively fixed by 31 July 2020.

\section{Quality assessment}

No standard checklist for assessing the quality of individual functional neuroimaging studies is available in previous studies. Based on related studies, we will employ an adapted checklist to assess included trial. ${ }^{17}$ Checklist is shown in box 1 .

\section{Data synthesis}

A table will be created to process extract data. We will calculate total and average sample size, age range of participants and patients' mean course of disease of the included studies. TMS parameters, functional neuroimaging and other significant clinical scales will be summarised. A quantitative, random-effects meta-analytic method known as activation likelihood estimation will be conducted to synthesis brain functional activity and connection changes after receiving 


\section{Box 1 Quality assessment of individual studies}

Category 1: sample characteristics (10)

1. Patients were evaluated with specific standardised diagnostic criteria (1).

2. Important demographic data (age and gender) were reported with mean (or median) and standard deviations (or range) (2).

3. Healthy comparison subjects were evaluated to exclude psychiatric and medical illnesses and demographic data were reported (2).

4. Important clinical variables (eg, depressive episodes, medication status and illness duration and severity, depression-related scale score) were reported with mean (or median) and standard deviations (or range) (3).

5. Sample size per group $>10$ (2).

Category 2: Methodology and reporting (8)

6. Magnet strength at least 1.5T (1).

7. At least 5 min of resting state acquisition (1).

8. Whole brain coverage of resting scans (1).

9. The acquisition and preprocessing techniques were clearly described so that they could be reproduced (1).

10. Coordinates reported in a standard space (1).

11. The parameters of TMS treatment (eg, frequency, intensity, duration, rate, time per session, pulses per condition and total stimulation sessions) are comprehensively provided (1).

12. Significant results are reported after correction for multiple testing using a standard statistical procedure (AlphaSim, FDR (False Discovery Rate), FWE (Family Wise Error) or permutation-based methods) (1).

13. Conclusions were consistent with the results obtained and the limitations were discussed (1).

TMS treatment for depression. ${ }^{18}{ }^{19}$ In order to clarify major factors underlying any high heterogeneity, subgroup analyses and meta-regression will be performed according to the placement, distance, frequency, rate, duration, time per session, pulses per condition and total stimulation sessions of TMS. A test of interaction will be used to address whether chance can explain the differences between subgroups. Also, moderation and mediation factor analysis will be conducted when performing subgroup analyses. We will attempt to adjust covariates before the analysis when they are not comparable across studies. Adjusting incomparable covariates after analysis will not be performed unless it is really necessary. All adjusting incomparable covariates will be illustrated. If modest heterogeneity exists, we will discuss the origin of the variation and whether it is methodological, clinical or statistical. We will conduct a jack-knife sensitivity analysis to test for study heterogeneity. This method assumes that those brain regions where the jackknife sensitivity analysis demonstrates significant difference are more replicable and robust. After excluding any unusual result, we will restart the meta-analysis or consider performing a multilevel meta-analysis. If heterogeneity is substantial, we might explore the sources of variation and trade the meta-analysis for a narrative review. As for publication bias, a funnel plot may be created according to effect size of any treatment-related changes in scores for depression to evaluate whether the findings are largely influenced by small studies, which in turn provides insight into publication bias or robustness against publication bias. ${ }^{20}$ Data synthesis and analysis will be completed by 20 August 2020 .

\section{PATIENT AND PUBLIC INVOLVEMENT}

This work will be based on published studies; therefore, patient or public involvement will be not required. Relevant results will be published in peer-reviewed journals.

\section{ETHICS AND DISSEMINATION}

Ethical approval is not required as primary data will not be collected. This review will be published in a peerreviewed journal.

Contributors BZ was responsible for this study. BZ, YD and WL conceived and designed the study. YD participated in drafting the protocol and preparing the manuscript. All authors read and approved the final manuscript.

Funding The authors have not declared a specific grant for this research from any funding agency in the public, commercial or not-for-profit sectors.

Competing interests None declared.

Patient and public involvement Patients and/or the public were not involved in the design, or conduct, or reporting, or dissemination plans of this research.

Patient consent for publication Not required.

Provenance and peer review Not commissioned; externally peer reviewed.

Open access This is an open access article distributed in accordance with the Creative Commons Attribution Non Commercial (CC BY-NC 4.0) license, which permits others to distribute, remix, adapt, build upon this work non-commercially, and license their derivative works on different terms, provided the original work is properly cited, appropriate credit is given, any changes made indicated, and the use is non-commercial. See: http://creativecommons.org/licenses/by-nc/4.0/.

ORCID iD

Bin Zhang http://orcid.org/0000-0002-9280-8247

\section{REFERENCES}

1 World Health Organization. Depression and other common mental disorders global health estimates. Geneva: World Health organization, 2017. Available: https://www.who.int/mental_health/ management/depression/prevalence_global_health_estimates/en/

2 Tibubos AN, Brähler E, Ernst M, et al. Course of depressive symptoms in men and women: differential effects of social, psychological, behavioral and somatic predictors. Sci Rep 2019;9:18929.

3 Porter RJ, Douglas KM. Cognitive impairment in people remitted from major depression. Lancet Psychiatry 2019;6:799-800.

4 Carpenter LL, Janicak PG, Aaronson ST, et al. Transcranial magnetic stimulation (TMS) for major depression: a multisite, naturalistic, observational study of acute treatment outcomes in clinical practice. Depress Anxiety 2012;29:587-96.

5 Blumberger DM, Vila-Rodriguez F, Thorpe KE, et al. Effectiveness of theta burst versus high-frequency repetitive transcranial magnetic stimulation in patients with depression (THREE-D): a randomised non-inferiority trial. Lancet 2018;391:1683-92.

6 Cantone M, Bramanti A, Lanza G, et al. Cortical plasticity in depression. ASN Neuro 2017;9:175909141771151.

7 Spampinato $\mathrm{C}$, Aguglia E, Concerto $\mathrm{C}$, et al. Transcranial magnetic stimulation in the assessment of motor cortex excitability and treatment of drug-resistant major depression. IEEE Trans Neural Syst Rehabil Eng 2013;21:391-403.

8 Ridding MC, Rothwell JC. Is there a future for therapeutic use of transcranial magnetic stimulation? Nat Rev Neurosci 2007;8:559-67.

9 Concerto C, Lanza G, Cantone M, et al. Repetitive transcranial magnetic stimulation in patients with drug-resistant major depression: a six-month clinical follow-up study. Int J Psychiatry Clin Pract 2015;19:252-8. 
10 Gershon AA, Dannon PN, Grunhaus L. Transcranial magnetic stimulation in the treatment of depression. Am J Psychiatry 2003;160:835-45.

11 Cash RFH, Cocchi L, Anderson R, et al. A multivariate neuroimaging biomarker of individual outcome to transcranial magnetic stimulation in depression. Hum Brain Mapp 2019;40:4618-29.

12 Downar J, Geraci J, Salomons TV, et al. Anhedonia and rewardcircuit connectivity distinguish nonresponders from responders to dorsomedial prefrontal repetitive transcranial magnetic stimulation in major depression. Biol Psychiatry 2014;76:176-85.

13 Fitzgerald PB, Maller JJ, Hoy KE, et al. Exploring the optimal site for the localization of dorsolateral prefrontal cortex in brain stimulation experiments. Brain Stimul 2009;2:234-7.

14 De Witte S, Klooster D, Dedoncker J, et al. Left prefrontal neuronavigated electrode localization in tDCS: 10-20 EEG system versus MRI-guided neuronavigation. Psychiatry Res 2018;274:1-6.

15 Moher D, Shamseer L, Clarke M, et al. Preferred reporting items for systematic review and meta-analysis protocols (PRISMA-P) 2015 statement. Syst Rev 2015;4:1.
16 Mutz J, Edgcumbe DR, Brunoni AR, et al. Efficacy and acceptability of non-invasive brain stimulation for the treatment of adult unipolar and bipolar depression: a systematic review and meta-analysis of randomised sham-controlled trials. Neurosci Biobehav Rev 2018;92:291-303.

17 Iwabuchi SJ, Krishnadas R, Li C, et al. Localized connectivity in depression: a meta-analysis of resting state functional imaging studies. Neurosci Biobehav Rev 2015;51:77-86.

18 Grace SA, Rossell SL, Heinrichs M, et al. Oxytocin and brain activity in humans: a systematic review and coordinate-based meta-analysis of functional MRI studies. Psychoneuroendocrinology 2018;96:6-24.

19 van Heeringen K, Bijttebier S, Desmyter S, et al. Is there a neuroanatomical basis of the vulnerability to suicidal behavior? A coordinate-based meta-analysis of structural and functional MRI studies. Front Hum Neurosci 2014;8:824.

20 Müller VI, Cieslik EC, Laird AR, et al. Ten simple rules for neuroimaging meta-analysis. Neurosci Biobehav Rev 2018;84:151-61. 